\title{
El uso de modelos matemáticos en evaluación económica de intervenciones de salud
}

\author{
MARIANELA CASTILLO-RIQUELME ${ }^{1, \mathrm{a}}$
}

'Departamento de Economía de la Salud, División de Planificación Sanitaria, Subsecretaría de Salud Pública, Ministerio de Salud de Chile. ${ }^{a}$ Economista en Salud, MSc in Health Management (Imperial College- London).

Correspondencia a: Marianela Castillo-Riquelme Monjitas 689, Santiago Centro, Fax: 0057 (2) 6384390 Email:marianela.castillo@ minsal.cl

\section{The use of mathematical modeling in economic evaluation of healthcare interventions}

The use of mathematical modeling in economic evaluation is a frequent practice seeking to inform priority setting in healthcare. The main advantages are the possibilities of extending the analyses of randomized controlled trails to specific population groups, and in routine conditions. Likewise, modeling allows transforming intermediate outcomes (such as subrogate markers) into final health outcomes. Models, help estimating the potential impact of an intervention in the population prior implementation, and for different scenarios. In the case of diseases with complex epidemiological characteristics, such as transmission dynamics of infectious diseases, models allow to reproduce these dynamics based on assumptions of the relationships among the variables. In this way, cost-effectiveness analysis of control interventions can be estimated. Additionally, models allow evaluating the uncertainty associated to the limitations of the available evidence, in a systematic, comprehensive and economic way. Nonetheless, the use of modeling is not free of criticisms, being the main ones related to the high number of assumptions and the luck of transparency of the internal processes (black box).

(Rev Med Chile 2010; 138 (Supl 2): 98-102).

Key words: Modeling, economic evaluation, decision analysis.

\section{RESUMEN}

La construcción y uso de modelos matemáticos en evaluación económica es una práctica frecuente que busca informar la toma de decisiones en salud. Dentro de sus principales ventajas se encuentra la posibilidad de extender el análisis o extrapolar los resultados de estudios clínicos aleatorizados en una población específica y en condiciones rutinarias. Asimismo, permiten simular resultados finales en salud a partir de resultados intermedios (ejemplo marcadores subrogados). Los modelos permiten estimar el impacto potencial de una intervención en diversos escenarios de implementación. En el caso de enfermedades cuya epidemiología y dinámica de transmisión es compleja, como enfermedades infecciosas, los modelos permiten reproducir la dinámica de transmisión basado en supuestos acerca de las interrelaciones entre sus variables. Se puede estimar así la costo-efectividad de intervenciones que alteran estas interrelaciones. Adicionalmente, los modelos permiten investigar la incertidumbre asociada a las limitaciones de la evidencia disponible en forma sistemática, integral y a costos bajos. Sin embargo, el uso de modelos en evaluación económica no está libre de críticas, siendo las principales asociadas al gran número de supuestos y la falta de transparencia de sus procesos e inferencias internas.

Palabras clave: Modelos, evaluación económica, análisis de decisiones 


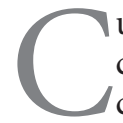
ualquier modelo busca ser una representación simple de una realidad normalmente compleja. Por ejemplo, un modelo econométrico permitirá evaluar la influencia de la edad y la condición social en el resultado clínico de una terapia. Philips et al se refieren a los modelos como un enfoque explícito para sintetizar la evidencia disponible sobre la efectividad y costos de intervenciones de salud mutuamente excluyentes ${ }^{1}$. Drummond et al destacan el rol de los modelos de análisis de decisiones para concentrar y sistematizar esta evidencia ${ }^{2}$. Los modelos en general, y en particular los de análisis de decisiones, tienen su origen en la Teoría de Probabilidades, y comparten fundamentos con la Teoría de Utilidad Esperada. Asimismo, el análisis de decisiones guarda directa relación con la Estadística Bayesiana en su forma de incorporar en el análisis cada tipo de evidencia y su incertidumbre asociada ${ }^{3,4}$. Los modelos buscan identificar el efecto que las diferentes variables e interrelaciones definidas entre éstas, tienen sobre los costos y outcomes de las intervenciones en evaluación.

\section{Las técnicas de modelamiento más usadas en evaluación económica}

Existen diferentes técnicas de modelamiento. Como puntualiza Gold et al, los modelos pueden ser clasificados de acuerdo a varias características no mutuamente excluyentes ${ }^{5}$. Por ejemplo, los modelos determinísticos son aquellos que usan el número promedio de eventos en la población, mientras que los modelos estocásticos usan un proceso de randomización para simular la distribución de probabilidades de los eventos que pueden ocurrir. Por otro lado, los modelos se pueden clasificar entre analíticos y de simulación. Los primeros son aquellos que desarrollan un proceso en el tiempo y dentro de esta categoría los árboles de decisiones y los modelos de estados de transición son los más usados. Los modelos Markov son definidos como un tipo especial de estado de transición, en el cual la probabilidad de transición depende solamente de estado actual. Los modelos también pueden ser clasificados en cohortes longitudinales (por ejemplo una cohorte homogénea, típicamente para análisis de decisiones) o de cohorte poblacional (para monitorear los estados de salud de una población en el tiempo $)^{5}$.
En un árbol de decisiones, las distintas opciones de intervenciones y sus respectivos cursos de acción son identificados y representados en distintas ramas del árbol. Cada rama se compone de eventos secuenciales que representan ya sea puntos de decisión (nodo cuadrado) o de resultado del evento previo (nodo circular). A todos los eventos que representan resultados se les asignan probabilidades, usando la mejor evidencia disponible. Además, los costos y las consecuencias (outcomes) asociados con cada nodo final se debe definir ya sea en base a la evidencia o a estudios primarios. Así, un árbol de decisiones es analizado usando el método estadístico llamado análisis inverso (folding back) y promediado. La Figura 1 ilustra un árbol de decisiones simplificado con dos alternativas de terapia (A y B), cada una con dos posibles resultados, así existen cuatro posibles nodos finales cada uno con una cierta probabilidad de observarse y con determinados costos y outcomes (QALYs en este caso) asociados. En este ejemplo, siguiendo el proceso de análisis inverso (multiplicando de derecha a izquierda los resultados por sus respectivas probabilidades), se descarta la alternativa A, ya que tiene menos utilidad y más costos esperados. Así la alternativa $B$ es la alternativa dominante, porque es a vez la más efectiva y la de menor costo. Sin embargo, no siempre en el análisis de costo efectividad se encuentra una alternativa dominante, es usual que exista una alternativa más efectiva que es a la vez más costosa.

Con los valores esperados de estas dos intervenciones se determina el indicador de costo

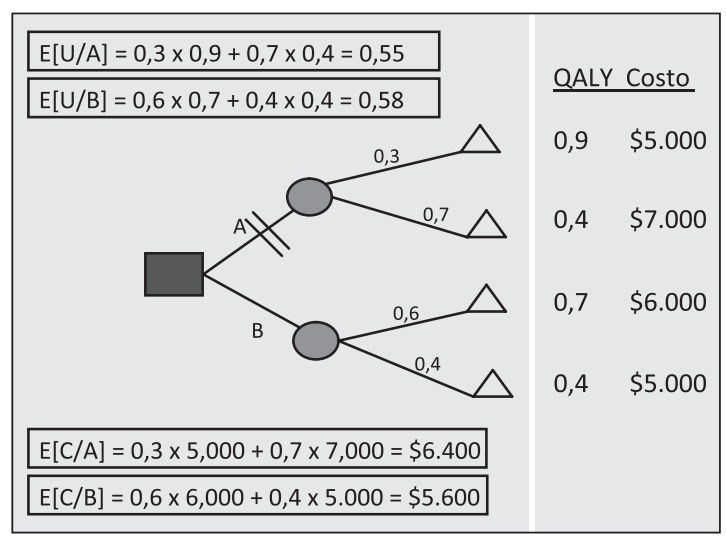

Figura 1. Árbol de decisiones con dos ramas y cuatro posibles desenlaces. 
efectividad incremental (ICER), el cual resume la comparación de dos intervenciones.

$$
\text { ICER }=\frac{E(C / B)-E(C / A)}{E(U / B)-E(U / A)}=\frac{5.600-6.400}{0,58-0,55}=-\frac{800}{0,03}=-26.667
$$

Para determinar el ICER se toma como base la intervención más efectiva, en este caso es B, así el ICER resulta en un ahorro de $\$ 26.667$ por cada QALY ganada.

La estructura de un árbol de decisiones puede resultar bastante compleja si se evalúan varias opciones que desencadenan en varios outcomes probables y cuando el desenlace se extiende por varios períodos de tiempo. Así, los árboles de decisiones son útiles cuando se trata de condiciones agudas que se dan sólo una vez o cuando la condición (aun crónica) se desarrolla en un período relativamente corto de tiempo, ver por ejemplo, Retinopatía de Prematurez en Castillo-Riquelme et $\mathrm{al}^{6}$.

Los modelos de estados de transición superan varias de las limitantes de un árbol de decisiones, especialmente en relación al horizonte temporal de evaluación. Estos modelos distribuyen miembros de una población (o cohorte) en un número finito de estados de salud y desde ahí, estos transitan entre estados en función de las probabilidades de transición definidas para un período. La duración de período de evaluación se denomina ciclo. Esta técnica se ha usado para estimar la esperanza de vida (o sobrevida) de distintos grupos de pacientes, ya que generalmente el modelo se evalúa hasta el punto en que todos los individuos llegan al es- tado de absorción, el cual corresponde a la muerte.

Los modelos de Markov son definidos como un tipo especial de estado de transición, en el cual la probabilidad de transición depende solamente del estado actual. Los modelos de Markov son útiles cuando el riesgo de la patología es contínuo en el tiempo (como es el caso de las varias enfermedades crónicas), cuando la temporalidad u oportunidad del evento es importante y cuando un evento puede ocurrir más de una vez ${ }^{7}$. En un modelo de Markov las probabilidades pueden variar con los ciclos, sin embargo, si las probabilidades de transición son constantes en el tiempo se habla de una cadena de markov. Los modelos de Markov no tienen memoria en relación a la historia de los pacientes. La Figura 2 muestra gráficamente un modelo de Markov simple, con tres estados de salud para un ciclo determinado.

Las flechas muestran la dirección de transición y su probabilidad. Cuando la flecha vuelve al mismo estado de salud, representa la probabilidad de permanencia en ese estado. La evaluación de distintas intervenciones es posible alterando la estructura del modelo, esto se refiere principalmente la definición de las probabilidades. Por ejemplo, la Figura 2 puede representar la historia natural de una enfermedad, la cual ante la existencia de un tratamiento efectivo reducirá las probabilidades de permanecer enfermo y de morir. En cada ciclo, la simulación computa costos y utilidades asociados a la permanencia del paciente o cohorte en los diferentes estados de salud, los cuales al final del horizonte de evaluación, representan el principal producto del modelo. Con los costos y outcomes se

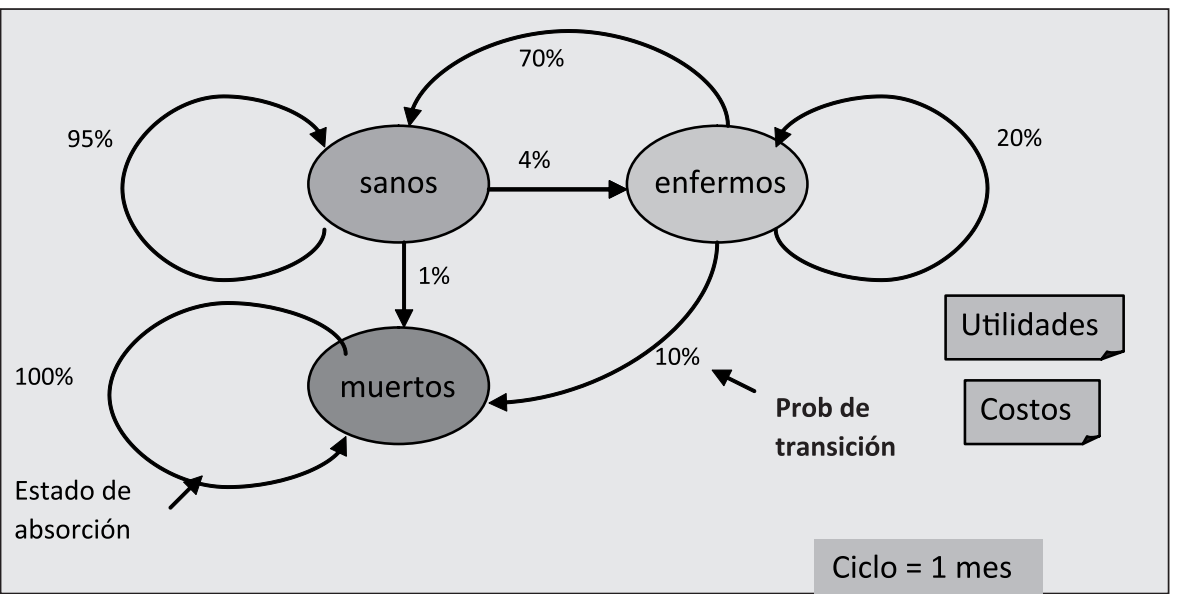

Figura 2. Representación gráfica de un ciclo Markov (3 estados). 
procede a calcular las razones de costo efectividad e ICER entre las distintas estrategias evaluadas.

Los datos de la Figura 1 se pueden escribir en una matriz de probabilidades como la que se muestra a continuación.

\begin{tabular}{|lccc|}
\hline $\begin{array}{l}\text { Probabilidad } \\
\text { de transición }\end{array}$ & $\begin{array}{c}\text { Hacia } \\
\text { Estado } \\
\text { "sanos" }\end{array}$ & $\begin{array}{c}\text { Estado } \\
\text { "enfermos" }\end{array}$ & $\begin{array}{c}\text { Estado } \\
\text { "muerte" }\end{array}$ \\
\hline $\begin{array}{l}\text { Estado } \\
\text { "sanos" }\end{array}$ & 0,95 & 0,04 & 0,01 \\
DE $\begin{array}{l}\text { Estado } \\
\text { "enfermos" }\end{array}$ & 0,7 & 0,2 & 0,1 \\
\hline $\begin{array}{l}\text { Estado } \\
\text { "muertos" }\end{array}$ & 0,0 & 0,0 & 1,0 \\
\hline
\end{tabular}

Las críticas a los modelos de Markov apuntan principalmente a su falta de memoria e incapacidad de relacionar la historia clínica previa de un paciente con su probabilidad de transición actual. Por ejemplo, se argumenta que el riesgo de tener un infarto cardíaco es distinto si el paciente ya tuvo uno.

Tanto los árboles de decisiones como los procesos Markov se pueden combinar para evaluar la simulación de un problema o estrategia de intervención.

Árboles con nodos Markov: Cuando el desenlace de un árbol de decisiones es muy complejo se puede conectar un proceso Markov, normalmente en un nodo final.

Ciclo Markov de árbol: Se usa un árbol de decisiones para representar o determinar la probabilidad de transición, además cada estado de salud puede estar modelado por un árbol de decisiones. Así se permite desagregar un gran problema en pequeños problemas, más manejables, los cual brinda más transparencia en problemas complejos y es más flexible para hacer cambios y conducir análisis de sensibilidad.

\section{Otros enfoques y clasificaciones de modelos}

Petitte, desde una perspectiva más amplia, se concentra en tres modelos para síntesis cuantitativa en medicina; meta-análisis, análisis de costo efectividad y análisis de decisión ${ }^{8}$. El propósito de un meta-análisis es combinar estudios previamente publicados para generar un resumen de conclusiones sobre un ámbito específico de investigación. Este método se usa para resumir evidencia previa en estudios clínicos aleatorizados, cuando los estudios son demasiado pequeños para constituir por sí mismos una conclusión válida ${ }^{8}$. Los datos obtenidos por este medio también pueden ser incorporados como parámetros de entrada en otros modelos, tales como árboles de decisiones, modelos de estado de transiciones, simulación Montecarlo o modelos de Markov.

\section{¿Cómo decidir o elegir qué modelo construir?}

Los factores que determinan la selección de un modelo específico son el objetivo del estudio, la naturaleza de la condición (su dinamismo) y la disponibilidad de datos. En palabras de Gold et $\mathrm{al}^{5}$. "En general es deseable usar el modelo más simple posible que capture el nivel de complejidad que es absolutamente necesario y para el cual hay datos disponibles para describir los parámetros del modelo" [página 156].

De acuerdo a Sculpher et $\mathrm{al}^{9}$, los modelos deben apoyar la toma decisiones racionales basada en la información existente, más que tratar de generar nueva información, hay que puntualizar que los modelos no sustituyen ensayos clínicos randomizados.

El avance tecnológico ha tenido un impacto muy favorable en el desarrollo de los modelos de decisión. Así las nuevas técnicas de microsimulación y de simulación de eventos discretos prometen solucionar varias de los problemas de los modelos analíticos tradicionales ${ }^{10}$.

\section{Reflexiones finales sobre el uso de modelos en evaluación económica}

Se debe elegir el modelo más simple que cumpla los requerimientos necesarios, hay que tener en cuenta que existe un trade-off entre la transparencia y la complejidad de los modelos, mientras más complejo menos transparente. Se debe propender a reducir el tamaño de la "caja negra", lo cual implica dejar todos los supuestos (parámetros y estructura) claramente definidos. La calidad de los inputs (solidez de la evidencia) y de 
la estructura definen la calidad de los outputs de un modelo. Se debe evaluar la consistencia del modelo a través de un proceso llamado calibración, el cual se puede medir a través de la capacidad de éste de simular óptimamente la situación actual o pasada. Los modelos no tienen capacidad de predicción del futuro, éstos son una proyección de lo que podría observarse bajo una serie de supuestos y condiciones. El análisis de sensibilidad es elemental para validar la solidez y representatividad de los resultados. A pesar de la existente controversia a nivel internacional, la mayoría de los investigadores recomienda el uso de análisis de sensibilidad probabilística sobre los parámetros de entrada. Sin embargo, recientes directrices ${ }^{1}$ diferencian entre cuatro fuentes de incertidumbre para las cuales se recomiendan acciones concretas de análisis. Estas son incertidumbre metodológica, estructural, de heterogeneidad y de parámetros. El nivel de incertidumbre capturado por un análisis de sensibilidad puede indicar la pertinencia de recolectar más información, así surge la noción de valor esperado de información perfecta ${ }^{4}$, el cual reporta el costo (por ejemplo en QALYs perdidas) de no contar con información adecuada al momento de tomar decisiones.

La aceptación de los modelos por parte de los que toman las decisiones, dependerá en gran parte de la facilidad para entenderlos ${ }^{10}$, de ahí la necesidad de explicar los resultados en forma resumida, en un lenguaje claro y sencillo. Cabe señalar que ningún modelo es perfecto, pero su contribución se debe evaluar en contraposición a la frecuente toma de decisiones (sobre adopción de tecnologías), sin elementos de análisis sistemático o, ante la dilatación de éstas decisiones por falta de información.

Finalmente, hay que señalar que existen diversas directrices elaboradas en un intento de homologar los métodos tanto para la construcción, selección y evaluación de evidencia, validación y el análisis de sensibilidad de los modelos para evaluación económica, como los reportados por Philips et al, ISPOR Task Force, y Sculpher et a ${ }^{1,11,9}$.

\section{Referencias}

1. Philips Z, Bojke L, Sculpher M, Claxton K, Golder S. Good Practice Guidelines for Decision-Analytic Modelling in Health Technology Assessment A Review and Consolidation of Quality Assessment, Pharmacoeconomics 2006; 24 (4): 355-71.

2. Drummond MF, Sculpher MJ, Torrance GW, O’Brien BJ, Stoddart GL. Methods for the Economic Evaluation of Health Care Programmes. 2005, Third edition, Oxford University Press [Capítulo 9: Economic evaluation using decision analytic modelling].

3. Bayesian Initiative in Health Economics \& Outcomes Research, A primer on Bayesian Statistics in Health Economics and Outcomes Research, MEDTAP International, Inc. 2003 (disponible en http://www.shef.ac.uk/ content/1/c6/02/55/92/primer.pdf).

4. Claxton K, Bayesian approaches to the value of information: implications for the regulation of new pharmaceuticals. Health Economics 1999; 8 (3): 269-74.

5. Gold MR, Siegel JE, Russell LB, Weinstein MC. (Eds) (1996) Cost-Effectiveness in Health and Medicine, Oxford University Press, New York. [Capítulo 5: Assessing the effectiveness of Health Interventions, páginas 151-63].

6. Castillo-Riquelme M, Lord J, Moseley M, Fielder A, Haines L. Cost-effectiveness of digital photographic screening for retinopathy of prematurity in the United Kingdom. International Journal of Technology Assessment in Health Care 2004; 20 (2): 201-13.

7. Sonnenberg FA, Beck JR, Markov Models in Medical Decision Making: A practical Guide. Medical Decision Making Vol 13 (4), Oct-Dec 1993.

8. Petitti D. (2000). Meta-Analysis, Decision Analysis and Cost-effectiveness Analysis. Methods for quantitative synthesis in medicine, Oxford University Press.

9. Sculpher M, Fenwick E, Claxton K. Assessing Quality in Decision Analytic Cost-Effectiveness Models. Pharmacoeconomics 2000 May; 17 (5): 461-77.

10. Weinstain MC. Resent Development in DecisionAnalytic Modelling for Economic Evaluation. Pharmacoeconomics 2006; 24 (11): 1043-53.

11. ISPOR Task Force. Principles of good practice for decision- analytic modeling in health care evaluation. Value Health 2003; 6: 9-17. 
